\title{
Fatigue Failure Model for Polymeric Compliant Systems
}

\author{
Theddeus T. Akano and Omotayo A. Fakinlede \\ Department of Systems Engineering, University of Lagos, Akoka, Lagos 101017, Nigeria \\ Correspondence should be addressed to Theddeus T. Akano; manthez@yahoo.com
}

Received 21 January 2013; Accepted 28 February 2013

Academic Editors: H. M. da Costa, A. Mousa, and A. Uygun

Copyright (C) 2013 T. T. Akano and O. A. Fakinlede. This is an open access article distributed under the Creative Commons Attribution License, which permits unrestricted use, distribution, and reproduction in any medium, provided the original work is properly cited.

\begin{abstract}
Fatigue analysis and lifetime evaluation are very important in the design of compliant mechanisms to ensure their safety and reliability. Earlier models for the fatigue prediction of compliant mechanisms are centred on repeated and reversed stress cycles. Compliant mechanisms (CMs) are now being applied to situations where the fatigue is caused by random varying stress cycles. It is, therefore, necessary to consider fatigue resulting from random varying stress cycles and damage caused to the compliant material. A continuum damage mechanics (CDM) model is proposed to assess the fatigue life of polymeric compliant mechanisms. The elastic strain energy is computed on the basis of a nearly incompressive hyperelastic constitution. The damage evolution equation is used to develop a mathematical formula that describes the fatigue life as a function of the nominal strain amplitude under cyclic loading. Low density polypropylene (LDP) is used for the fatigue tests conducted under displacement controlled condition with a sine waveform of $10 \mathrm{~Hz}$. The results from the theoretical formula are compared with those from the experiment and fatigue software. The result from the prediction formula shows a strong agreement with the experimental and simulation results.
\end{abstract}

\section{Introduction}

Fatigue is one of the major failure mechanisms in engineering structures [1]. Time-varying cyclic loads result in failure of components at stress values below the yield or ultimate strength of the material. Fatigue failure of components takes place by the initiation and propagation of a crack until it becomes unstable and then propagates to sudden failure. The total fatigue life is the sum of crack initiation life and crack propagation life. Fatigue life prediction has become important because of the complex nature of fatigue as it is influenced by several factors, statistical nature of fatigue phenomena and time-consuming fatigue tests.

Though a lot of fatigue models have been developed and used to solve fatigue problems, the range of validity of these models is not well defined. No method would predict the fatigue life with the damage value by separating crack initiation and propagation phases. The methods used to predict crack initiation life are mainly empirical [2] and they fail to define the damage caused to the material. Stress- or strainbased approaches followed do not specify the damage caused to the material, as they are mainly curve fitting methods. The limitation of this approach motivated the development of micromechanics models termed as local approaches based on continuum damage mechanics (CDM). The local approaches are based on application of micromechanics models of fracture in which stress/strain and damage at the crack tip are related to the critical conditions required for fracture. These models are calibrated through material specific parameters. Once these parameters are derived for particular material, they can be assumed to be independent of geometry and loading mode and may be used to the assessment of a component fabricated from the same material.

For some compliant structures, the desired motion may occur infrequently, and the static theories may be enough for the analysis [3]. However, by the definition of compliant mechanisms, deflection of flexible members is required for the motion. Usually, it is desired that the mechanism be capable of undergoing the motion many times, and design requirements may be many millions of cycle of infinite life. This repeated loading cause fluctuating stresses in the members and can result in fatigue failure. Failure can occur at stresses that are significantly lower than those that cause static failure [3]. A small crack is enough to initiate the fatigue failure. The crack progresses rapidly since the stress concentration effect becomes greater around it. If the stressed 
area decreases in size, the stress increases in magnitude, and if the remaining area is small, the member can fail. A member failed because of fatigue showing two distinct regions. The first one is due to the progressive development of the crack, while the other one is due to the sudden fracture. Premature or unexpected failure of a device can result in an unsafe design. The consumer confidence may be reduced in products that fail prematurely. For these and other reasons, it is critical that the fatigue life of compliant mechanism be analyzed.

Although fatigue failure is difficult to predict accurately, an understanding of fatigue failure prediction and prevention is very helpful in the design of compliant mechanisms. The theory can be used to design devices that will withstand these fluctuating stresses.

Several models are available for fatigue failure prediction. The stress-life and strain-life models are commonly used in the design of mechanical components [3]. These theories are appropriate for parts that undergo consistent and predictable fluctuating stresses. Many machine components fit into this category because their motion and loads are defined by kinematics of the mechanism. There are three stress cycles with which loads may be applied to the component under consideration. The simplest being the reversed stress cycle (Figure 1(a)). This is merely a sine wave where the maximum stress and minimum stress differ by a negative sign. An example of this type of stress cycle would be in an axle, in which every half turn or half period as in the case of the sine wave, the stress on a point would be reversed. The most common type of cycle found in engineering applications is where the maximum stress and minimum stress are asymmetric (the curve is a sine wave), not equal, and opposite (Figure 1(b)). This type of stress cycle is called repeated stress cycle. A final type of cycle mode is where stress and frequency vary randomly (Figure 1(c)). An example of this would be hull shocks, where the frequency magnitude of the waves will produce varying minimum and maximum stresses.

Predicting the life of parts stressed above the endurance limit is at best a rough procedure. For the large percentage of mechanical and structural compliant systems subjected to randomly varying stress cycle intensity (e.g., compliant automotive suspension and compliant aircraft structural components, etc.), the prediction of fatigue life is further complicated. The normal stress-life and strain-life models cannot be adopted in the fatigue prediction. Models such as continuum damage mechanics (CDM) can be used in dealing with this situation.

Polymers are predominantly used in the design of compliant mechanisms [3]. It is important to use the nonlinear characteristics of polymers to analyse the performance of compliant systems. Thermoplastic polymers like polypropylene exhibit a viscoelastic material response [4]. It has been frequently noted that with certain constitutive laws, such as those of viscoelasticity and associative plasticity, the material behaves in a nearly incompressible manner [5]. The typical volumetric behavior of hyperelastic materials can be grouped into two classes. Materials such as polymers typically have small volumetric changes during deformation and those that are incompressible or nearly-incompressible materials [6]. An example of the second class of materials is foams, which can experience large volumetric changes during deformation, and these are compressible materials. This implies that most polymers are nearly incompressible. In general, the response of a typical polymer is strongly dependent on temperature [7]. At low temperatures, polymers deform elastically like glass; at high temperatures, the behaviour is viscous like liquids; at moderate temperatures, the behaviour is like a rubbery solid. Hyperelastic constitutive laws are intended to approximate this rubbery behaviour. Polymers are capable of large deformations and subject to tensile and compression stress-strain curves [8]. The simplest yet relatively precise description for this type of material is isotropic hyperelasticity [8].

The fatigue failure of thermoplastics polymers generally develops in two phases [9]. First, the material accumulates fatigue damage (i.e., in the initiation phase), which ultimately leads to the formation of visible crazes. The crazes further grow, form cracks and propagate (i.e., in the propagation phase) until the final failure occurs. In general, the damage process in polymers is regarded as the formation and development of microdefects and crazes within an initially perfect material. The material remains the same but its macroscopic properties change with its microscopic geometry [10]. In polymers, craze formation is generally believed to be one of the main causes of material damage, which is both a localized yielding process and the first stage of fracture. Crazes are usually initiated either at surface flaws and scratches or at internal voids and inclusions and affect significantly the subsequent deformation and bulk mechanical behaviors of polymers [11]. The continuum damage mechanics (CDM) first introduced by Kachanov and developed within the framework of thermodynamics discusses systematically the effects of microdefects on the subsequent development of microdefects and the states of stress and strain in materials. It has been applied to fatigue and fracture of different materials.

In this paper, an isotropic damage evolution equation for finite viscoelasticity characteristic of polymeric CMs is proposed, which is based on the CDM. A new damage model is developed to establish the fatigue life formula for such compliant systems. The compliant material is idealized as an isotropic hyperelastic material. A commonly used polymeric material, low density polypropylene (LDP) was tested to obtain the fatigue life as a function of the strain amplitude.

\section{The Literature Review}

A few researchers have looked into the fatigue failure of compliant systems. Li et al. [12] used the modified Basquin equation to determine the life cycle till failure for compliant fast tool servo. The fatigue life according to the equation is a function of the equivalent reverse stress, fatigue stress concentration factor, the range stress, the ultimate strength, the average stress, and the endurance limit. Demirel et al. [13] and Subaşi [14] used the factor of safety expressed in terms of the fluctuating stresses, endurance limit, mean stress component, and an alternating stress component for fatigue failure prediction of compliant mechanism. If the stress condition is below the two lines described in modified 


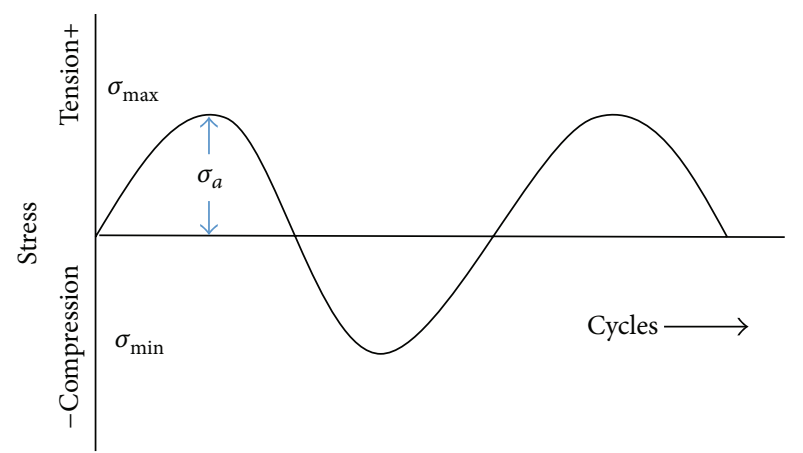

(a) Reversed

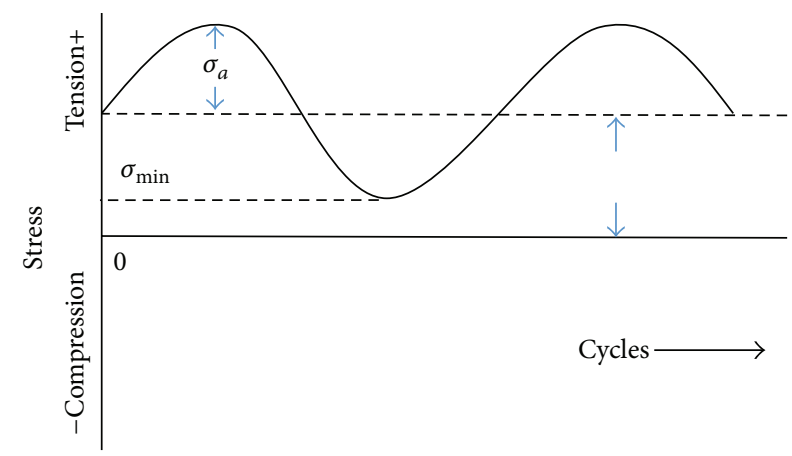

(b) Repeated

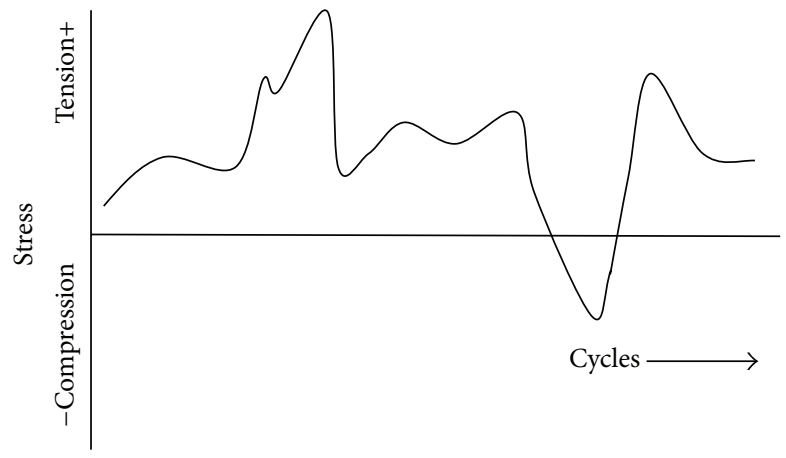

(c) Random

FIGURE 1: Stress cycles showing (a) reversed, (b) repeated, and (c) random cycles.

Goodman diagram for fatigue failure, the compliant member is expected to have an infinite life. Howell et al. [15] proposed a method for the probabilistic design of a bistable compliant slider-crank mechanism which its objective function is the maximization of the mechanism reliability in fatigue. Cannon et al. [16] used the modified fatigue strength at cycles which is expressed in terms of Marin correction factors and the theoretical fatigue strength to predict the failure behaviour of a compliant end-effector for microscribing. This gives the $\mathrm{S}-\mathrm{N}$ diagram for the mechanism where the maximum stress is compared with the modified fatigue strength.

Quite a number of researchers have employed the concept of damage evolution in the prediction of fatigue failure of engineering structures and components. Jiang [17] derived a damaged evolution model for strain fatigue of ductile metals based on Lemaitre's potential of dissipation. Then the equation of fatigue life prediction and the criterion of cumulative fatigue damage were deduced. The model was validated with experiment. Shi et al. [18] proposed a new damage mechanics model to predict the fatigue life of fiber reinforced polymer lamina and adopted the singularity of stiffness matrix as the failure criterion of lamina in this article, which inventively transformed the complex anisotropic issue of composite lamina fatigue into the analysis of singlevariable isotropic damages for fiber and matrix. Akshantala and Talreja [19] proposed a methodology for fatigue-life prediction that utilizes a micromechanics-based evaluation of damage evolution in conjunction with a semiempirical fatigue failure criterion. The specific case treated was that of crossply laminates under cyclic tension. The predicted results were compared with experimental data for several glass epoxy and carbon epoxy laminates. Ping et al. [20] proposed a nonlinear continuum damage mechanics model to assess the creep-fatigue life of a steam turbine rotor, in which the effects of complex multiaxial stress and the coupling of fatigue and creep are taken into account. The results were compared with those from the linear accumulation theory that had been dominant in life assessment of steam turbine rotors. The comparison shows that the nonlinear continuum damage mechanics model describes the accumulation and development of damage better than the linear accumulation theory. Ali et al. [21] investigated the fatigue behaviour of rubber using dumb-bell test specimens under uniaxial loading. In modeling fatigue damage behaviour, a continuum damage model was presented based on the function of the strain range under cyclic loading. Upadhyaya and Sridhara [22] predicted strain controlled fatigue life of EN 19 steel and 6082-T6 aluminum alloy considering both crack initiation and crack propagation phases. The theory of continuum damage mechanics was used in the study of fatigue damage phenomena such as the nucleation and initial defect growth (microvoids and microcracks) in elastomers by Wang et al. [23] and Mahmoud et al. [24].

Continuum damage mechanics (CDM) approach models the crack initiation life with a damage value, and damage beyond the crack initiation phase is predicted by fracture mechanics in terms of crack size. Fatigue life was predicted based on this concept. 


\section{Fatigue Failure Prediction Model}

Finite element implementations of nearly incompressible material models often employ decoupled numerical treatments of the dilatation and deviatoric parts of the deformation gradient. The strain energy density function for such material is decoupled as

$$
\Psi(C)=\Psi(\bar{C})+U(J),
$$

where

$$
\begin{gathered}
\Psi(\overline{\mathbf{C}})=\mu_{p}(\operatorname{tr} \mathbf{C}-3), \\
\mathrm{U}(J)=\beta(J-1)^{2}, \\
\mu_{p}=\frac{1}{2} \sum_{i=1}^{n} \mu_{i} ; \quad \beta=\frac{k_{i}}{2},
\end{gathered}
$$

$k$ and $\mu$ are the material properties known as bulk and shear modulus, respectively; $J$ is the Jacobian determinant of the deformation gradient. The strain energy density could be expressed in terms of the principal stretches $\lambda_{j}$ as

$$
\Psi\left(\lambda_{1}, \lambda_{2}, \lambda_{3}\right)=\sum_{p=1}^{n} \frac{\mu_{p}}{\alpha_{p}}\left(\bar{\lambda}_{1}^{\alpha_{p}}+\bar{\lambda}_{2}^{\alpha_{p}}+\bar{\lambda}_{3}^{\alpha_{p}}-3\right)+\sum_{p=1}^{n} \beta(J-1)^{2} .
$$

The principal components of the Cauchy stress are given by [25]

$$
\sigma_{i}=\frac{\lambda_{i}}{\lambda_{1} \lambda_{2} \lambda_{3}} \frac{\partial \Psi}{\partial \lambda_{1}}, \quad i=1,2,3
$$

The strain energy potential can be written as either function of the principal stretch ratios or as a function of the invariants of the strain tensor $C, I_{1}, I_{2}, I_{3}$. The invariants of $\mathrm{C}$ are defined as

$$
\begin{gathered}
I_{1}=\operatorname{tr} \mathbf{C}=\mathbf{C}: \mathbf{I}, \\
I_{2}=\operatorname{tr} \mathbf{C C}, \\
I_{3}=\operatorname{det} \mathbf{C}=J^{2} .
\end{gathered}
$$

In terms of the principal stretch ratio, the invariants are written as

$$
\begin{gathered}
I_{1}=\lambda_{1}^{2}+\lambda_{2}^{2}+\lambda_{3}^{2}, \\
I_{2}=\lambda_{1}^{2} \lambda_{2}^{2}+\lambda_{2}^{2} \lambda_{3}^{2}+\lambda_{3}^{2} \lambda_{1}^{2}, \\
I_{2}=\lambda_{1}^{2} \lambda_{2}^{2} \lambda_{3}^{2} .
\end{gathered}
$$

The invariants could be expressed in terms of the deviatoric principal stretches as

$$
\begin{gathered}
\bar{I}_{1}=J^{-2 / 3} I_{1}, \\
\bar{I}_{2}=J^{-4 / 3} I_{2}, \\
\bar{I}_{3}=J^{2} .
\end{gathered}
$$

Substituting (6) and (8) into (1) gives

$$
\Psi=\sum_{p=1}^{n} \frac{\mu_{p}}{\alpha_{p}}\left(J^{-2 / 3}\left(\lambda_{1}^{\alpha_{p}}+\lambda_{2}^{\alpha_{p}}+\lambda_{3}^{\alpha_{p}}\right)-3\right)+\sum_{p=1}^{n} \beta(J-1)^{2} .
$$

Substituting (10) into (4) gives

$$
\begin{aligned}
\lambda_{i} \frac{\partial J}{\partial \lambda_{i}}=\sum_{p=1}^{n} \frac{\mu_{p}}{\alpha_{p}}( & -\frac{2}{3} J^{-5 / 3} \lambda_{i} \frac{\partial J}{\partial \lambda_{i}}\left(\lambda_{1}^{\alpha_{p}}+\lambda_{2}^{\alpha_{p}}+\lambda_{3}^{\alpha_{p}}\right) \\
& \left.+J^{-2 / 3} \alpha_{p} \lambda_{i}^{\alpha_{p}}\right) \\
+ & \sum_{p=1}^{n} 2 \beta(J-1) \lambda_{i} \frac{\partial J}{\partial \lambda_{i}} .
\end{aligned}
$$

Since $J=\lambda_{1}^{2} \lambda_{2}^{2} \lambda_{3}^{2}$, we have

$$
\lambda_{i} \frac{\partial J}{\partial \lambda_{i}}=\lambda_{1}^{2} \lambda_{2}^{2} \lambda_{3}^{2}=J
$$

Therefore,

$$
\begin{aligned}
\lambda_{i} \frac{\partial \Psi}{\partial \lambda_{i}}=\left[\sum_{p=1}^{n} \frac{\mu_{p}}{\alpha_{p}} J^{-2 / 3}\left(\alpha_{p} \lambda_{i}^{\alpha_{p}}-\frac{2}{3}\left(\lambda_{1}^{\alpha_{p}}+\lambda_{2}^{\alpha_{p}}+\lambda_{3}^{\alpha_{p}}\right)\right)\right. \\
\left.+\sum_{p=1}^{n} 2 \beta J(J-1)\right] .
\end{aligned}
$$

The principal Cauchy stresses are, therefore, given as

$$
\begin{aligned}
\sigma_{i}=\left[\sum_{p=1}^{n} \frac{\mu_{p}}{\alpha_{p}} J^{-5 / 3}\left(\alpha_{p} \lambda_{i}^{\alpha_{p}}-\frac{2}{3}\left(\lambda_{1}^{\alpha_{p}}+\lambda_{2}^{\alpha_{p}}+\lambda_{3}^{\alpha_{p}}\right)\right)\right. \\
\left.+\sum_{p=1}^{n} 2 \beta J(J-1)\right] .
\end{aligned}
$$

Then, the difference between the principal stresses becomes

$$
\begin{aligned}
& \sigma_{1}-\sigma_{3}=\sum_{p=1}^{n} \mu_{p} J^{-5 / 3}\left(\lambda_{1}^{\alpha_{p}}-\lambda_{3}^{\alpha_{p}}\right), \\
& \sigma_{2}-\sigma_{3}=\sum_{p=1}^{n} \mu_{p} J^{-5 / 3}\left(\lambda_{2}^{\alpha_{p}}-\lambda_{3}^{\alpha_{p}}\right), \\
& \sigma_{1}-\sigma_{2}=\sum_{p=1}^{n} \mu_{p} J^{-5 / 3}\left(\lambda_{1}^{\alpha_{p}}-\lambda_{2}^{\alpha_{p}}\right) .
\end{aligned}
$$

Since force is applied in one direction in most compliant mechanisms, we will consider a mechanism undergoing uniaxial stress state. The principal stretches become

$$
\lambda_{1}=\lambda ; \quad \lambda_{2}=\lambda_{3}=\sqrt{\frac{J}{\lambda}}
$$


$\lambda$ is the stretch in the loading direction; $\lambda_{2}$ and $\lambda_{3}$ are the principal stretches on plane perpendicular to loading direction. Substituting (16) into (15) gives

$$
\begin{gathered}
\sigma_{1}-\sigma_{3}=\sigma_{1}-\sigma_{2}=\sum_{p=1}^{n} \mu_{p} J^{-5 / 3}\left(\lambda^{\alpha_{p}}-\left(\frac{J}{\lambda}\right)^{\alpha_{p} / 2}\right), \\
\sigma_{2}-\sigma_{3}=0 .
\end{gathered}
$$

The effective stress is given by the equation tensor

$$
\begin{aligned}
\sigma_{e} & =\left(\frac{1}{2}\left(\sigma_{1}-\sigma_{2}\right)^{2}+\left(\sigma_{2}-\sigma_{3}\right)^{2}+\left(\sigma_{1}-\sigma_{3}\right)^{2}\right)^{1 / 2} \\
& =\left(\frac{3}{2} S_{i j} S_{i j}\right)^{1 / 2},
\end{aligned}
$$

where $S_{i j}$ are the components of the deviator tensor $\sigma_{\mathrm{dev}}$

$$
\sigma_{\mathrm{dev}}=\sigma-\frac{1}{3}(\operatorname{tr} \boldsymbol{\sigma}) I .
$$

Substituting (17) into (19) gives

$$
\sigma_{e}=\sum_{p=1}^{n} \mu_{p} J^{-5 / 3}\left(\lambda^{\alpha_{p}}-\left(\frac{J}{\lambda}\right)^{\alpha_{p} / 2}\right) .
$$

The stretch ratio in the loading direction is given by [26]

$$
\lambda=1+\varepsilon,
$$

$\varepsilon$ is the nominal strain. Substituting (21) into (20) gives

$$
\sigma_{e}=\sum_{p=1}^{n} \mu_{p} J^{-5 / 3}\left((1+\varepsilon)^{\alpha_{p}}-\left(\frac{J}{(1+\varepsilon)}\right)^{\alpha_{p} / 2}\right) .
$$

Substituting (16) into (10), we have

$$
\begin{aligned}
\Psi= & \sum_{p=1}^{n} \frac{\mu_{p}}{\alpha_{p}}\left(J^{-2 / 3}\left(\lambda^{\alpha_{p}}+2\left(\frac{J}{\lambda}\right)^{\alpha_{p} / 2}\right)-3\right)+\sum_{p=1}^{n} \beta(J-1) \\
= & \sum_{p=1}^{n} \frac{\mu_{p}}{\alpha_{p}}\left(J^{-2 / 3}\left((1+\varepsilon)^{\alpha_{p}}+2\left(\frac{J}{(1+\varepsilon)}\right)^{\alpha_{p} / 2}\right)-3\right) \\
& +\sum_{p=1}^{n} \beta(J-1) .
\end{aligned}
$$

3.1. Continuum Damage Mechanics Model. Material damage usually induces the stiffness change of the material. Therefore, the damage state can be characterized by the change of elastic constants. Consider a representative volume element of an anisotropic material with stiffness $[E]$ damaged under a system of loading $\left\{\sigma_{e}\right\}$. The stiffness matrix of the damaged material is $\left[E_{d}\right]$. Then, the damage matrix $[D]$ is defined as [27]

$$
[D]=[I]-\left[E_{d}\right][E]^{-1},
$$

where $[I]$ is the identity matrix. The strain $\{\varepsilon\}$ is given as

$$
\begin{aligned}
\{\varepsilon\} & =[I]-\left[E_{d}\right]^{-1}\left\{\sigma_{e}\right\} \\
& =[E]^{-1}[[I]-[D]]^{-1}\left\{\sigma_{e}\right\} \\
& =[E]^{-1}\left\{\bar{\sigma}_{e}\right\},
\end{aligned}
$$

where

$$
\left\{\bar{\sigma}_{e}\right\}=[[I]-[D]]^{-1}\left\{\sigma_{e}\right\} .
$$

The matrix $\left\{\bar{\sigma}_{e}\right\}$ is defined as the effective stress matrix after material damage. Hence, the damage effect matrix $[M]$ is

$$
[M]=[[I]-[D]]^{-1} .
$$

3.1.1. Isotropic Model. Consider a damaged body as shown in Figure 2, in which a representative volume element (RVE) is isolated. Damaged variable is physically defined by the surface density of microcracks and intersections of microvoids lying on a plane cutting RVE of cross-section $\delta A$ [28]. Damaged variable $D_{(\bar{n})}$, for the plane defined by normal $\bar{n}$, is

$$
D_{(\bar{n})}=\frac{\delta A_{D}}{\delta A}, \quad 0 \leq D_{(\bar{n})} \leq 1,
$$

where $\delta A_{D}$ is the effective area of the intersection of all microcavities or microcracks that lie in the initial area $\delta A$ at time $t$. An isotropic damage variable is equally distributed in all directions, which is defined as

$$
D=\frac{\delta A_{D}}{\delta A} .
$$

Equation (29) is the percentage of the damaged area to initial area. $D$ is a scalar. Isotropic damage is assumed in this concept of continuum damage mechanics. The damage parameter can be obtained by reducing the rank of matrix to zero. This reduces (24) to

$$
D=1-E_{d} \cdot E^{-1} .
$$

The effective stress after material damage, $\bar{\sigma}_{e}$, and damage strain energy release rate are related by [29]

$$
\bar{\sigma}_{e}=M \cdot \sigma_{e}=\frac{\sigma_{e}}{1-D} .
$$

The nominal stress-strain relation of a damage material is the same in form as that of an undamaged material [30]. This means that

$$
\bar{\sigma}_{e}=\sigma_{e}
$$

so that

$$
\frac{\sigma_{e}}{1-D}=\sum_{p=1}^{n} \mu_{p} J^{-5 / 3}\left(\lambda^{\alpha_{p}}-J^{\alpha_{p} / 2} \lambda^{-\alpha_{p} / 2}\right) .
$$




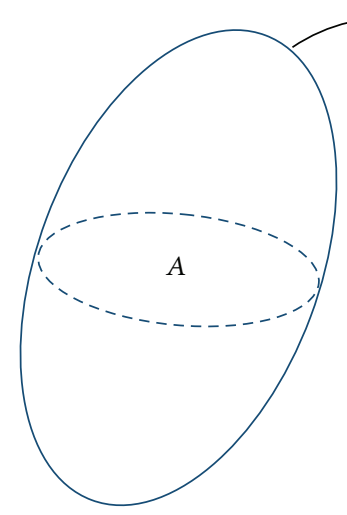

(a)

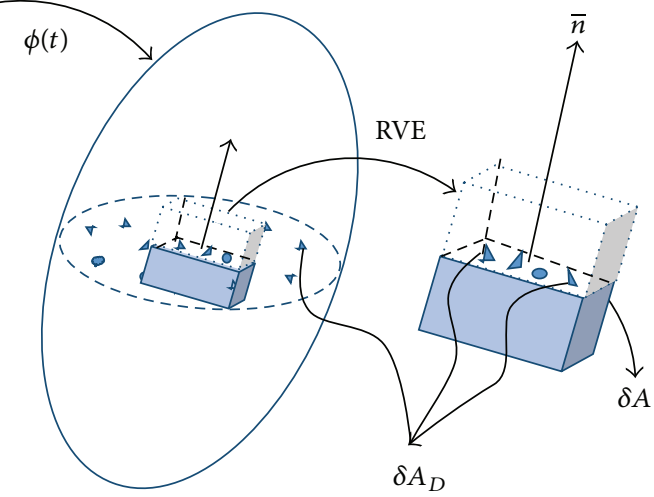

(b)

FIGURE 2: Transformation $\phi(t)$ from the (a) initial undamaged configuration to (b) the damaged configuration.

The constitutive equation for damage evolution is given by [28]

$$
\dot{D}=-\frac{\partial \alpha_{0}}{\partial \mathcal{Y}},
$$

$\alpha_{0}$ is the dissipation potential; $\mathcal{Y}$ is the damage strain energy release rate; $\dot{D}$ is the damage growth rate. The dissipation potential is assumed as [17]

$$
\alpha_{0}=\frac{s_{0}}{q_{0}+1}\left(\frac{-y}{s_{0}}\right)^{q_{0}+1} \text {, }
$$

$q_{0}$ and $s_{0}$ are material parameters determined by the experimental fatigue life as a function of the strain range. But the strain energy of a damaged and undamaged material is the same [27]. From the CDM theory, the damaged strain energy should be a function of the effective nominal normal stress of the damaged configuration. Hence, in the uniaxial stress state, the damaged strain energy released rate $\mathcal{Y}$ is defined as

$$
-\mathcal{Y}=\frac{\partial \Psi}{\partial D}=\frac{\partial \Psi\left(\bar{\sigma}_{e}\right)}{\partial D} .
$$

Substituting (23) into (36), we have

$$
\begin{aligned}
-\mathscr{Y} & =\frac{\partial \Psi\left(\bar{\sigma}_{e}\right)}{\partial D}=\frac{\partial \Psi}{\partial \lambda} \frac{\partial \lambda\left(\bar{\sigma}_{e}\right)}{\partial D} \\
& =\sum_{p=1}^{n} \frac{\mu_{p}}{\alpha_{p}} J^{-2 / 3}\left(\alpha_{p} \lambda^{\alpha_{p}-1}-\alpha_{p} J^{\alpha_{p} / 2} \lambda^{-\left(\alpha_{p} / 2+1\right)}\right) \frac{\partial \lambda\left(\bar{\sigma}_{e}\right)}{\partial D} .
\end{aligned}
$$

Taking the partial derivative of (33) with respect to $D$, gives

$$
\begin{aligned}
& \frac{\partial \lambda\left(\bar{\sigma}_{e}\right)}{\partial D} \\
& \quad=\sum_{p=1}^{n} \frac{1}{\mu_{p}} \frac{\sigma_{e}}{(1-D)^{2}}\left[J^{-5 / 3}\left(\alpha_{p} \lambda^{\alpha_{p}-1}+\frac{\alpha_{p}}{2} J^{\alpha_{p} / 2} \lambda^{-\left(\alpha_{p} / 2+1\right)}\right)\right]^{-1} .
\end{aligned}
$$

Substituting (38) into (37), we have

$$
\begin{aligned}
-\mathscr{Y}=\sum_{p=1}^{n} \frac{1}{\alpha_{p}} & \frac{\sigma_{e}}{(1-D)^{2}} J \\
\times & {\left[\left(\alpha_{p} \lambda^{\alpha_{p}-1}-\alpha_{p} J^{\alpha_{p} / 2} \lambda^{-\left(\alpha_{p} / 2+1\right)}\right)\right.} \\
& \left.\times\left(\alpha_{p} \lambda^{\alpha_{p}-1}+\frac{\alpha_{p}}{2} J^{\alpha_{p} / 2} \lambda^{-\left(\alpha_{p} / 2+1\right)}\right)^{-1}\right] .
\end{aligned}
$$

Substituting (33) into (39), we have

$$
\begin{aligned}
-\mathscr{Y}=\sum_{p=1}^{n} \frac{\mu_{p}}{\alpha_{p}}[ & \frac{\left(\lambda^{\alpha_{p}}-J^{\alpha_{p} / 2} \lambda^{-\alpha_{p} / 2}\right)}{(1-D)} J^{-2 / 3} \\
& \times\left[\left(\alpha_{p} \lambda^{\alpha_{p}-1}-\alpha_{p} J^{\alpha_{p} / 2} \lambda^{-\left(\alpha_{p} / 2+1\right)}\right)\right. \\
& \left.\left.\times\left(\alpha_{p} \lambda^{\alpha_{p}-1}+\frac{\alpha_{p}}{2} J^{\alpha_{p} / 2} \lambda^{-\left(\alpha_{p} / 2+1\right)}\right)^{-1}\right]\right] .
\end{aligned}
$$

Using (34), (35), and (40), we have

$$
\begin{aligned}
\dot{D}=\sum_{p=1}^{n} \frac{\mu_{p}}{\alpha_{p}}\left(s_{0}^{-1}\right)[ & \frac{\left(\lambda^{\alpha_{p}}-J^{\alpha_{p} / 2} \lambda^{-\alpha_{p} / 2}\right)}{(1-D)} J^{-2 / 3} \\
\times & {\left[\left(\alpha_{p} \lambda^{\alpha_{p}-1}-\alpha_{p} J^{\alpha_{p} / 2} \lambda^{-\left(\alpha_{p} / 2+1\right)}\right)\right.} \\
& \left.\left.\times\left(\alpha_{p} \lambda^{\alpha_{p}-1}+\frac{\alpha_{p}}{2} J^{\alpha_{p} / 2} \lambda^{-\left(\alpha_{p} / 2+1\right)}\right)^{-1}\right]\right]^{q_{0}}
\end{aligned}
$$

Under a cyclic loading condition, the damage will accumulate with the number of cycles, and the damage evolution will depend on the strain amplitude. The time rate change of 
damage variable $\dot{D}$ can be represented in terms of the evolution of $D$ with respect to the number of cycles. Based on this consideration, the principal stretch amplitude $\Delta \lambda$ is used to replace $\lambda$, the fatigue damage evolution per cycle is then expressed as

$\frac{d D}{d N}$

$$
\begin{aligned}
=\sum_{p=1}^{n} \frac{\mu_{p}}{\alpha_{p}}\left(s_{0}^{-1}\right)[ & \frac{\left(\Delta \lambda^{\alpha_{p}}-J^{\alpha_{p} / 2} \Delta \lambda^{-\alpha_{p} / 2}\right)}{(1-D)} J^{-2 / 3} \\
\times & {\left[\left(\alpha_{p} \Delta \lambda^{\alpha_{p}-1}-\alpha_{p} J^{\alpha_{p} / 2} \Delta \lambda^{-\left(\alpha_{p} / 2+1\right)}\right)\right.} \\
& \left.\left.\times\left(\alpha_{p} \Delta \lambda^{\alpha_{p}-1}+\frac{\alpha_{p}}{2} J^{\alpha_{p} / 2} \Delta \lambda^{-\left(\alpha_{p} / 2+1\right)}\right)^{-1}\right]\right]^{q_{0}},
\end{aligned}
$$

where $\Delta \lambda$ is the principal stretch amplitude and $N$ is the number of cycles. Assuming that the damage variable $D$ is zero at the beginning of the cyclic loading, that is, $D=0$ when $N=0$, then the damage value at any cycle can be determined by integrating (42), which gives

$$
\begin{aligned}
& \int_{0}^{D}(1-D)^{q_{0}} d D \\
&=\int_{0}^{N}\left[\sum_{p=1}^{n} \frac{\mu_{p}}{\alpha_{p}}\left(s_{0}^{-1}\right) J^{-2 / 3}\right. \\
& \times \\
& \times\left[\left(\Delta \lambda^{\alpha_{p}}-J^{\alpha_{p} / 2} \Delta \lambda^{-\alpha_{p} / 2}\right)\right. \\
& \quad \times\left(\alpha_{p} \Delta \lambda^{\alpha_{p}-1}-\alpha_{p} J^{\alpha_{p} / 2} \Delta \lambda^{-\left(\alpha_{p} / 2+1\right)}\right) \\
&\left.\times\left(\alpha_{p} \Delta \lambda^{\alpha_{p}-1}+\frac{\alpha_{p}}{2} J^{\alpha_{p} / 2} \Delta \lambda^{-\left(\alpha_{p} / 2+1\right)}\right)^{-1}\right] d N .
\end{aligned}
$$

The relation between the damage variable $D$ and number of cycles $N$ could be deduced as

$$
\begin{aligned}
& {\left[1-(1-D)^{q_{0}+1}\right]} \\
& =\left(q_{0}+1\right) \\
& \times\left[\sum_{p=1}^{n} \frac{\mu_{p}}{\alpha_{p}}\left(s_{0}^{-1}\right) J^{-2 / 3}\right. \\
& \times\left[\left(\Delta \lambda^{\alpha_{p}}-J^{\alpha_{p} / 2} \Delta \lambda^{-\alpha_{p} / 2}\right)\right. \\
& \quad \times\left(\alpha_{p} \Delta \lambda^{\alpha_{p}-1}-\alpha_{p} J^{\alpha_{p} / 2} \Delta \lambda^{-\left(\alpha_{p} / 2+1\right)}\right) \\
& \left.\quad \times\left(\alpha_{p} \Delta \lambda^{\alpha_{p}-1}+\frac{\alpha_{p}}{2} J^{\alpha_{p} / 2} \Delta \lambda^{-\left(\alpha_{p} / 2+1\right)}\right)^{-1}\right]
\end{aligned} .
$$

The damage variable is expressed as the ratio of the number of cycles $N$ to the fatigue life $N_{f}$ as [17]

$$
D=\frac{N}{N_{f}} .
$$

Equation (45) indicates that the damage is linearly distributed to each cycle during the loading. Therefore, if a material has been subjected to cyclic loading, the damage is

$$
D=\frac{N_{i}}{\left(N_{f}\right)_{i}},
$$

and when the fatigue rupture occurs, we have

$$
D=D_{c}=1,
$$

$D_{c}$ is the critical value of the damage variable. Equations (45) to (47) show that at the moment of failure

$$
N=N_{f}
$$

and the fatigue life $N_{f}$ is expressed as

$$
\begin{aligned}
& N_{f}=\left(q_{0}+1\right)^{-1} \\
& \times\left[\sum_{p=1}^{n} \frac{\mu_{p}}{\alpha_{p}}\left(s_{0}^{-1}\right)\right. \\
& \times\left[\left(\Delta \lambda^{\alpha_{p}}-J^{\alpha_{p} / 2} \Delta \lambda^{-\alpha_{p} / 2}\right)\right. \\
& \quad \times\left(\alpha_{p} \Delta \lambda^{\alpha_{p}-1}-\alpha_{p} J^{\alpha_{p} / 2} \Delta \lambda^{-\left(\alpha_{p} / 2+1\right)}\right) \\
&\left.\left.\quad \times\left(\alpha_{p} \Delta \lambda^{\alpha_{p}-1}+\frac{\alpha_{p}}{2} J^{\alpha_{p} / 2} \Delta \lambda^{-\left(\alpha_{p} / 2+1\right)}\right)^{-1}\right]\right]^{-q_{0}}
\end{aligned}
$$

Using (21), the principal stretch amplitude, $\Delta \lambda$, and the nominal strain amplitude, $\Delta \varepsilon$, are related as

$$
\Delta \lambda=1+\Delta \varepsilon
$$

Substituting (50) into (49), the formula for the fatigue life is expressed as a function of the nominal strain amplitude for a compliant mechanism under large deformation and cyclic loading

$$
\begin{aligned}
& N_{f} \\
& =\left(q_{0}+1\right)^{-1}\left[\sum_{p=1}^{n} \frac{\mu_{p}}{\alpha_{p}}\left(s_{0}^{-1}\right)\right. \\
& \times\left[\left((1+\Delta \varepsilon)^{\alpha_{p}}-J^{\alpha_{p} / 2}(1+\Delta \varepsilon)^{-\alpha_{p} / 2}\right)\right. \\
& \times\left(\alpha_{p}(1+\Delta \varepsilon)^{\alpha_{p}-1}\right. \\
& \left.\quad-\alpha_{p} J^{\alpha_{p} / 2}(1+\Delta \varepsilon)^{-\left(\alpha_{p} / 2+1\right)}\right)
\end{aligned}
$$




$$
\begin{aligned}
& \times\left(\alpha_{p}(1+\Delta \varepsilon)^{\alpha_{p}-1}\right. \\
& \left.\left.\left.\quad+\frac{\alpha_{p}}{2} J^{\alpha_{p} / 2}(1+\Delta \varepsilon)^{-\left(\alpha_{p} / 2+1\right)}\right)^{-1}\right]\right]^{-q_{0}}
\end{aligned}
$$

If $n=1 ; \alpha=2$; and $J=1$, (51) reduces to the fatigue life $N_{f}$ for a Neo-Hookean incompressible model

$$
\begin{aligned}
N_{f}=\left(q_{0}+1\right)^{-1}\left[\frac{\mu_{p}}{2}\left(s_{0}^{-1}\right)\right. \\
\times\left[\left((1+\Delta \varepsilon)^{2}-(1+\Delta \varepsilon)^{-1}\right)\right. \\
\times 2\left((1+\Delta \varepsilon)-(1+\Delta \varepsilon)^{-2}\right) \\
\left.\left.\times\left(2(1+\Delta \varepsilon)+(1+\Delta \varepsilon)^{-2}\right)^{-1}\right]\right]^{-q_{0}} .
\end{aligned}
$$

Based on the experimental results and curve fitting, $q_{0}$ and $s_{0}$ are obtained as 5.54 and $6.83 \mathrm{MPa}$, respectively.

\section{Simulation and Experimental Test}

Testing is an important part of designing components for acceptable fatigue life. The analysis in the design phase is used to obtain dimensions that are most likely to provide desired results and to minimize costly and time-consuming iterations in prototyping and testing. Types of testing range form standard fatigue specimen tests to testing the actual device under operating conditions.

The testing of the actual device usually requires more work than for a standard test specimen. Two major challenges accompany the testing of compliant mechanisms. First, a new test must be designed for each new type of device. Secondly, because of the large number of cycles required for fatigue testing, the test device may also fail due to fatigue. Standard fatigue specimen test is adopted here.

4.1. Sample Preparation. Low density polypropylene was selected as the test material. The geometry and test length of the specimens are as shown in Figure 3. It has a gauge length of $22 \mathrm{~mm}$.

4.2. Finite Element Analysis (FEA). In order to better understand the stress distributions, FEA was performed. An FEA model of an undamaged LDP specimen was employed. The model was built in solid edge ST 4. For the static FEA, ANSYS 13.0 was used as pre/post processor and solver. The specimen model was subjected to static and cyclic loading. Then, ANSYS nCode DesignLife was used for the fatigue life analysis.

4.3. Fatigue and Uniaxial Tensile Tests. The BOSE ElectroForce (ELF) 3200 testing machine (Figure 4), in conjuction with the WinTest control software, was used to conduct the

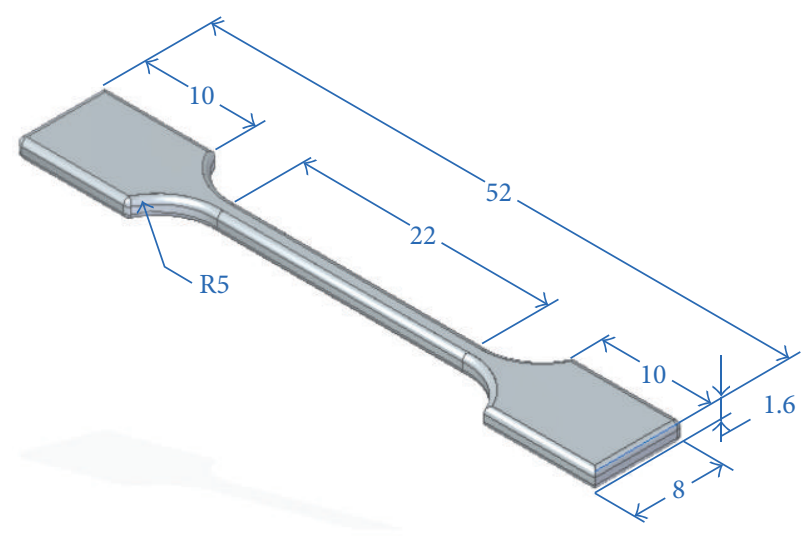

Figure 3: Geometry of LDP test specimen.

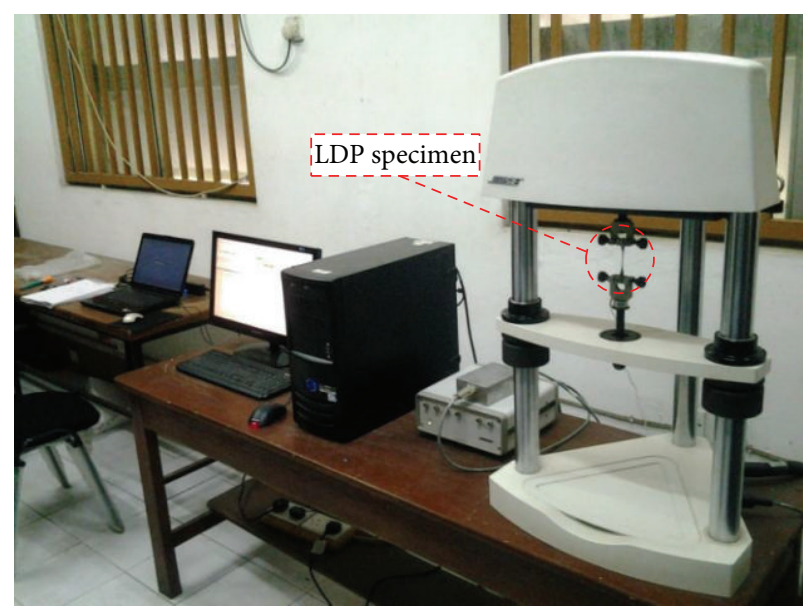

FIgURE 4: Experimental setup.

mechanical experiments in uniaxial cyclic loading and uniaxial tension. The test instrument incorporate proprietary Bose linear motion technologies and WinTest controls to provide a revolutionary approach to mechanical fatigue and dynamic characterization. The ELF has a maximum load of $225 \mathrm{~N}$ and a maximum frequency of $400 \mathrm{~Hz}$. A set of low mass grips, model GRP-TCDMA450N from BOSE ElectroForce (Eden Prairie, MN, USA), were used. The load cell had a maximum load rating of $2.5 \mathrm{~N}(250 \mathrm{~g})$ and resolution of $\pm 10 \mathrm{mg}$. The ELF 3200 measures displacements via a Capacitec $100 \mathrm{~lm}$ displacement transducer (model HPC-40/4101) used as a feedback for the control loop.

For the quasistatic tests, the specimens were placed under tension at a controlled rate of displacement $(0.02 \mathrm{~mm} / \mathrm{s})$. The clamping length was about $40 \mathrm{~mm}$. The mechanical properties, that is, Young's modulus, shear modulus, ultimate strain, and stress were assessed for the specimens. The average of the results was taken as the resultant value. To determine local data (stress and strain) from global data (force and displacement), the specimen's dimensions were obtained with the use of the micrometer screw gauge and the vernier caliper, and its modulus is calculated from the linear part of the resulting stress-strain curve shown in Figure 5. The material 


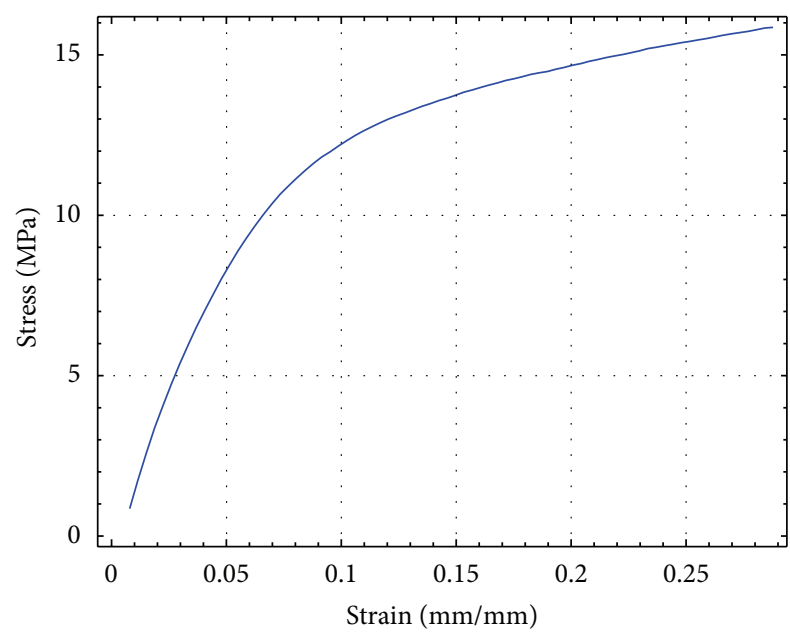

FIGURE 5: Stress-strain curve for LDP.

parameter $\mu_{p}$ is obtained by fitting the experimental stressstrain curve into (22). This yields $\mu_{p}=43.04 \mathrm{MPa}$.

The fatigue experiments were conducted between a minimum and maximum load in tension for a prescribed number of cycles using a set frequency. All fatigue tests were conducted at $10 \mathrm{~Hz}$. The residual strain was determined by considering the displacement reading on the oscilloscope at the initiation of the test and at the conclusion of the dwell phase. The residual strain was measured by subtracting the oscilloscope displacement value at the initiation of the test from the displacement at the conclusion of the constant-stress amplitude fatigue phase, once the specimen was unloaded to zero stress and allowed to dwell for a short time.

Approximately 40 fibers were tested in this investigation.

Approximately $20 \%$ of the fibers broke at the grip interface at the conclusion of the uniaxial tensile loading phase, which indicated premature failure due to a stress concentration near the grip interface. For this reason, these experimental results were omitted from the results in this study. All fibers were subjected to the same frequency during cyclic loading $(10 \mathrm{~Hz})$, elongation rate during uniaxial tension $(0.02 \mathrm{~mm} / \mathrm{s})$.

Figure 6 is the oscilloscope output of load and displacement versus time response of the LDP samples, while Figures 7 and 8 display their $1 \mathrm{~s}$ interval of the strain versus time and stress versus time response under sinusoidal cyclic loading conditions after equilibrium stress and strain values were reached. These samples were subjected to uniaxial fatigue loading conditions. The graphs show the behaviour of the samples under different displacement inputs. The strain range increases within increase in the input displacement. The fatigue life verses strain amplitude in a logarithmic coordinate is shown in Figure 9. The scattered points represent experimental results, the black dotted line is the result from fatigue simulation software while the red dotted line is from the derived formula.

4.4. Hysteresis Loop. In fatigue, the area of a stress-strain hysteresis loop is a measure of mechanical energy lost due

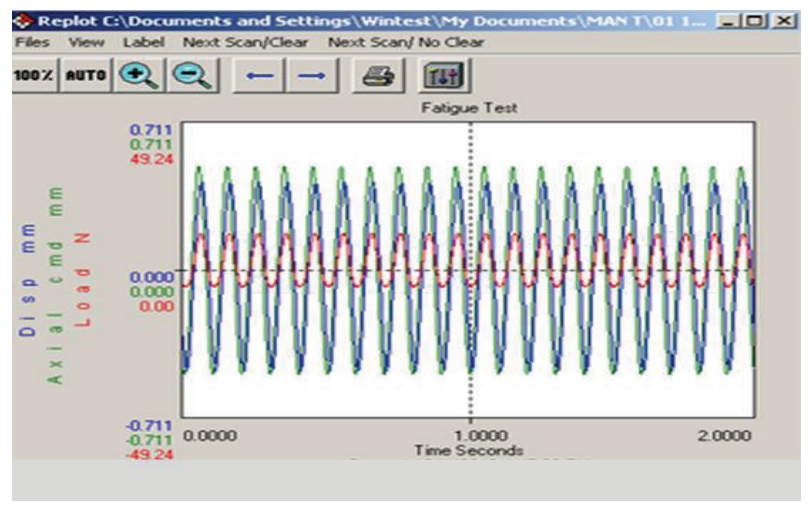

FIGURE 6: Oscilloscope output of load and displacement versus time response of LDP sample undergoing uniaxial sinusoidal loading.

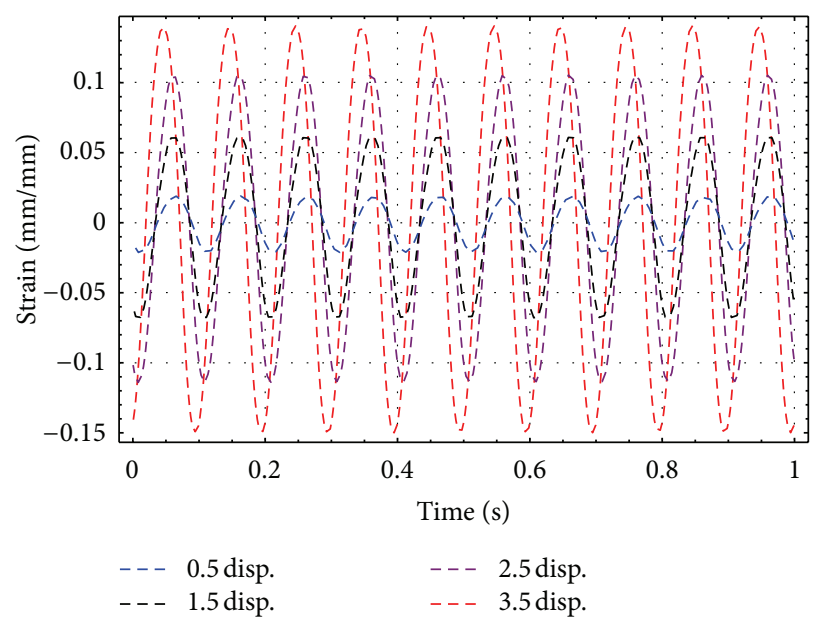

FIGURE 7: A second strain cycles.

to viscoelastic damping during each cycle of extension and compression. For a viscoelastic material subject to the cyclic loading, the hysteresis of the material can be defined by plotting the input stress $\sigma(t)$ versus the responding strain $\varepsilon(t)$ for one cycle of motion. Polymeric material hysteresis loops are difficult to analyse but can reveal interesting insight into the behaviour of the material during fatigue testing [31]. Figure 10 shows hysteresis loops of the low density polypropylene for 10 complete cycles. From the figure, the hysteresis curves for LDP are generally asymmetrical. The graph shows an early increase in maximum stress with increase in the input displacement load before it got to the maximum value and started decreasing. The loops had immediate stability for all range of displacement load input. The area captured within the hysteresis loop is equal to the dissipation energy per cycle of harmonic motion by the material. Within the tensile portion of the loop, considerable plastic strain and crack propagation energy is lost as the intersection through the zero stress line is at a positive strain value. During compression, the intersection of the zero stress line is very close to zero, implying little plastic strain. This behaviour of the material in compression reduces the amount of energy lost per cycle. 


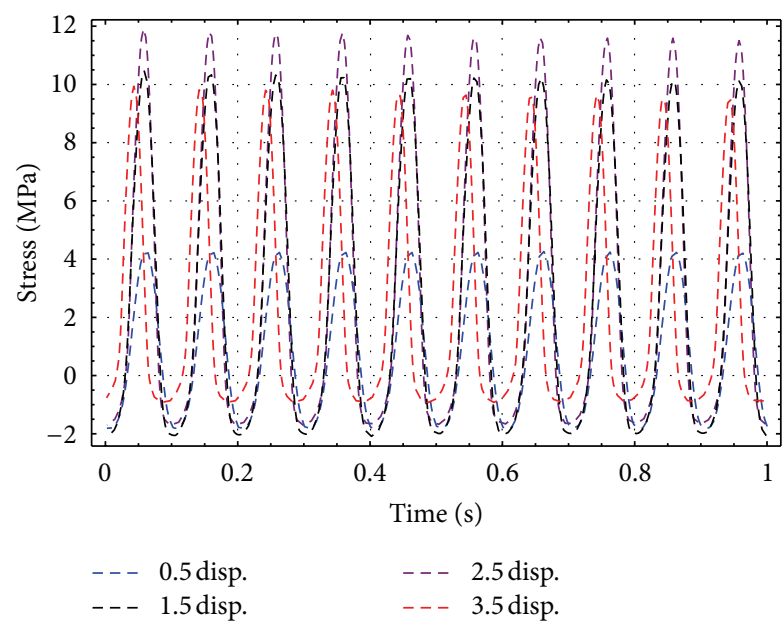

Figure 8: A second stress cycles.

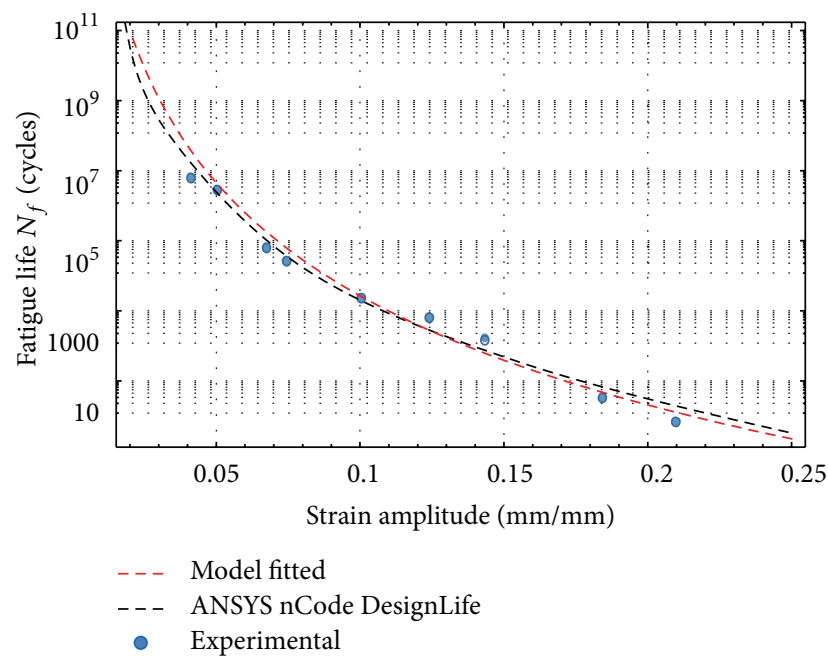

Figure 9: Fatigue life $N_{f}$ versus strain amplitude.

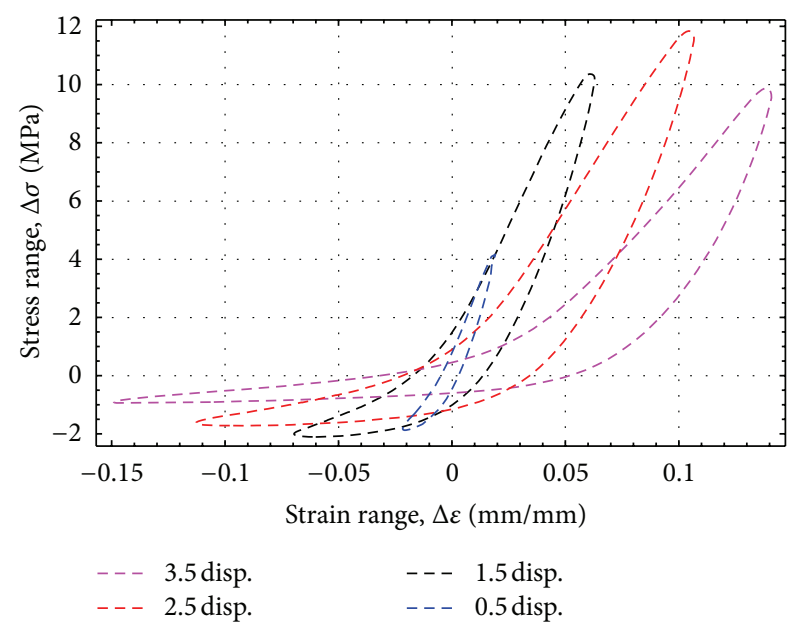

FIGURE 10: Hysteresis loop for the LDP material.

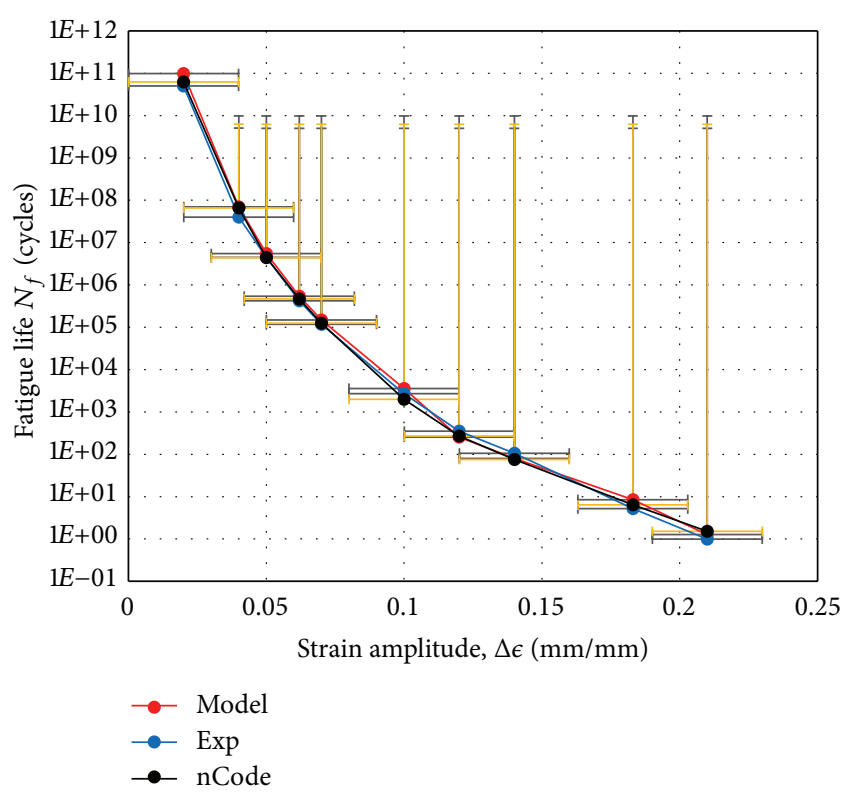

FIGURE 11: Standard error bars.

4.5. Statistical Inference Using Error Bars. Means and standard error (SE) bars are shown in Figure 11 for test experiments where the fatigue life in ten independent fatigue test experiments as well as the corresponding model and ANSYS nCode results of the test specimens were determined over strain amplitude (Figure 9). Error bars were used to assess differences between groups at the same strain amplitude points. Overlap rule was used to estimate the group points. The mean and their error regions overlapped, showing that the groups are not statistically and significantly different from one another.

\section{Conclusion}

A theoretical formula based on nominal strain amplitude for the fatigue life prediction of compliant polymeric material has been presented. The fatigue life prediction formula was developed within the context of continuum damage mechanics. Hyperelastic model is used to capture the large deformation behaviour of polymeric compliant mechanism. The strain energy function is formulated in terms of the strain invariants.

ANSYS nCode DesignLife, that is, mainly based on the reversed stress cycle, is also used to perform the fatigue simulation. Fatigue and tensile tests were conducted under displacement controlled loading condition. Nominal stressstrain curve was obtained from which the material parameters were determined by curve fitting.

Immediate stability in the hysteresis loops and little plastic damage that occurs within the compressive region of the fatigue cycles make LDP suitable for CMs.

The statistical analysis of the theoretical prediction formula with the experimental data and simulation result shows a strong agreement. Therefore, the CDM-based model can 
be applied to study fatigue life for compliant systems with varying stress and frequency.

\section{Conflict of Interests}

The authors declare that they have no conflict of interests.

\section{References}

[1] W. Schutz, "A history of fatigue," Engineering Fracture Mechanics, vol. 54, no. 2, pp. 263-300, 1996.

[2] Q. Y. Wang, J. Y. Berard, S. Rathery, and C. Bathias, "High-cycle fatigue crack initiation and propagation behaviour of highstrength spring steel wires," Fatigue and Fracture of Engineering Materials and Structures, vol. 22, no. 8, pp. 673-677, 1999.

[3] L. I. Howell, Compliant Mechanisms, John Wiley \& Sons, New York, NY, USA, 2001.

[4] N. D. Mankame and G. K. Ananthasuresh, "A novel compliant mechanism for converting reciprocating translation into enclosing curved paths," Journal of Mechanical Design, vol. 126, no. 4, pp. 667-672, 2004.

[5] O. C. Zienkiewicz and R. L. Taylor, The Finite Element Method, Butterworth-Heinemann, Boston, Mass, USA, 5th edition, 2000.

[6] ANSYS Documentation, 2012, http://www.kxcad.net/ansys/ ANSYS/ansyshelp/.

[7] A. F. Bower, Applied Mechanics of Solids, CRC Press, New York, NY, USA, 2010.

[8] X. Y. Gong and R. Moe, "On stress analysis for a hyperelastic material," in Proceedings of the ANSYS Conference, 2002.

[9] X. Li, H. A. Hristov, A. F. Yee, and D. W. Gidley, "Influence of cyclic fatigue on the mechanical properties of amorphous polycarbonate," Polymer, vol. 36, no. 4, pp. 759-765, 1995.

[10] C. Y. Tang, W. H. Tai, and W. B. Lee, "Modeling of damage behaviors of high impact polystyrene," Engineering Fracture Mechanics, vol. 55, no. 4, pp. 583-591, 1996.

[11] E. Passaglia, "Crazes and fracture in polymers," Journal of Physics and Chemistry of Solids, vol. 48, no. 11, pp. 1075-1100, 1987.

[12] H. Li, R. Ibrahim, and K. Cheng, "Design and principles of an innovative compliant fast tool servo for precision engineering," Mechanical Science, vol. 2, pp. 139-146, 2011.

[13] B. Demirel, M. T. Emirler, Ü. Sönmez, and A. Yörükoğlu, "Semicompliant force generator mechanism design for a required impact and contact forces," Journal of Mechanisms and Robotics, vol. 2, no. 4, Article ID 045001, 11 pages, 2010.

[14] L. Subaşi, Synthesis of compliant bistable four-link mechanisms for two positions [Thesis], Middle East Technical University, 2005.

[15] L. L. Howell, S. S. Rao, and A. Midha, "Reliability-based optimal design of a bistable compliant mechanism," Journal of Mechanical Design, vol. 116, no. 4, pp. 1115-1122, 1994.

[16] B. R. Cannon, T. D. Lillian, S. P. Magleby, L. L. Howell, and M. R. Linford, "A compliant end-effector for microscribing," Precision Engineering, vol. 29, no. 1, pp. 86-94, 2005.

[17] M. Jiang, "A damaged evolution model for strain fatigue of ductile metals," Engineering Fracture Mechanics, vol. 52, no. 6, pp. 971-975, 1995.
[18] W. Shi, W. Hu, M. Zhang, and Q. Meng, "A damage mechanics model for fatigue life prediction of fiber reinforced polymer composite lamina," Acta Mechanica Solida Sinica, vol. 24, no. 5, pp. 399-410, 2011.

[19] N. V. Akshantala and R. Talreja, "A micromechanics based model for predicting fatigue life of composite laminates," Materials Science and Engineering A, vol. 285, no. 1-2, pp. 303313, 2000.

[20] J. J. Ping, M. Guang, S. Yi, and X. SongBo, "An Effective continuum damage mechanics model for creep-fatigue life assessment of a steam turbine rotor," International Journal of Pressure Vessels and Piping, vol. 80, no. 6, pp. 389-396, 2003.

[21] A. Ali, M. Hosseini, and B. Sahari, "Continuum damage mechanics modeling for fatigue life of elastomeric materials," International Journal of Structural Integrity, vol. 1, no. 1, pp. 6372, 2010.

[22] Y. S. Upadhyaya and B. K. Sridhara, "Fatigue crack initiation and propagation life prediction of materials," in Proceedings of the International Conference on Mechanical, Electronics and Mechatronics Engineering (ICMEME '12), Bangkok, Thailand, 2012.

[23] B. Wang, H. Lu, and G. Kim, "A damage model for the fatigue life of elastomeric materials," Mechanics of Materials, vol. 34, no. 8, pp. 475-483, 2002.

[24] W. E. Mahmoud, S. A. Mansour, M. Hafez, and M. A. Salam, "On the degradation and stability of high abrasion furnace black (HAF)/acrylonitrile butadiene rubber (NBR) and high abrasion furnace black (HAF)/graphite/acrylonitrile butadiene rubber (NBR) under cyclic stress-strain," Polymer Degradation and Stability, vol. 92, no. 11, pp. 2011-2015, 2007.

[25] R. W. Ogden, Non-Linear Elastic Deformations, Dover, New York, NY, USA, 1997.

[26] H. W. Haslach and R. W. Armstrong, Deformable Bodies and Their Material Behavior, John Wiley \& Sons, New York, NY, USA, 2004.

[27] C. Y. Tang and W. B. Lee, "Damage mechanics applied to elastic properties of polymers," Engineering Fracture Mechanics, vol. 52, no. 4, pp. 717-729, 1995.

[28] J. Lemaitre and R. Desmorat, Engineering Damage Mechanics, Springer, Berlin, Germany, 2005.

[29] J. Lemaitre, "A continuum damage mechanics model for ductile fracture," Journal of Engineering Materials and Technology, vol. 107, no. 1, pp. 83-89, 1985.

[30] F. Sidoroff, "Description of anisotropic damage application to elasticity," in IUTAM Colloquium on Physical Non-Linearities in Structures, J. Hult and J. Lemaitre, Eds., pp. 237-244, Springer, Berlin, Germany, 1981.

[31] N. Fern, P. Alam, F. Touaiti, and M. Toivakka, "Fatigue life predictions of porous composite paper coatings," International Journal of Fatigue, vol. 38, pp. 181-187, 2012. 

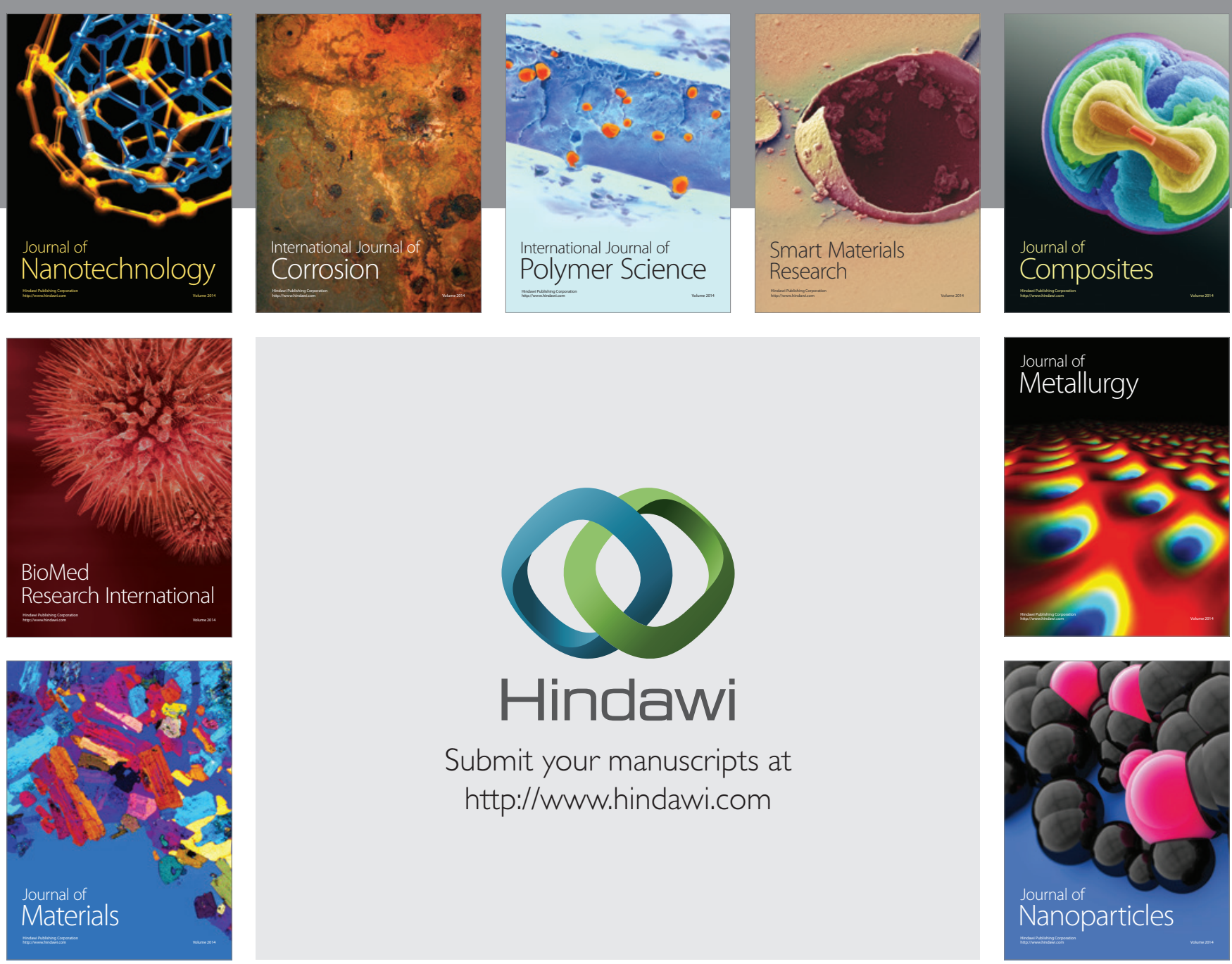

Submit your manuscripts at http://www.hindawi.com
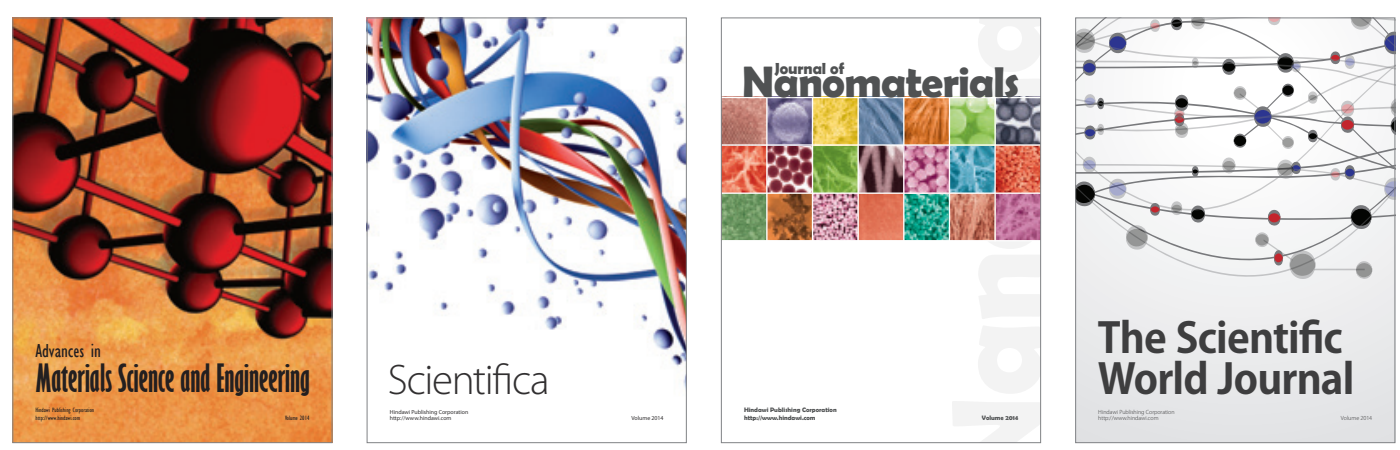

\section{The Scientific World Journal}
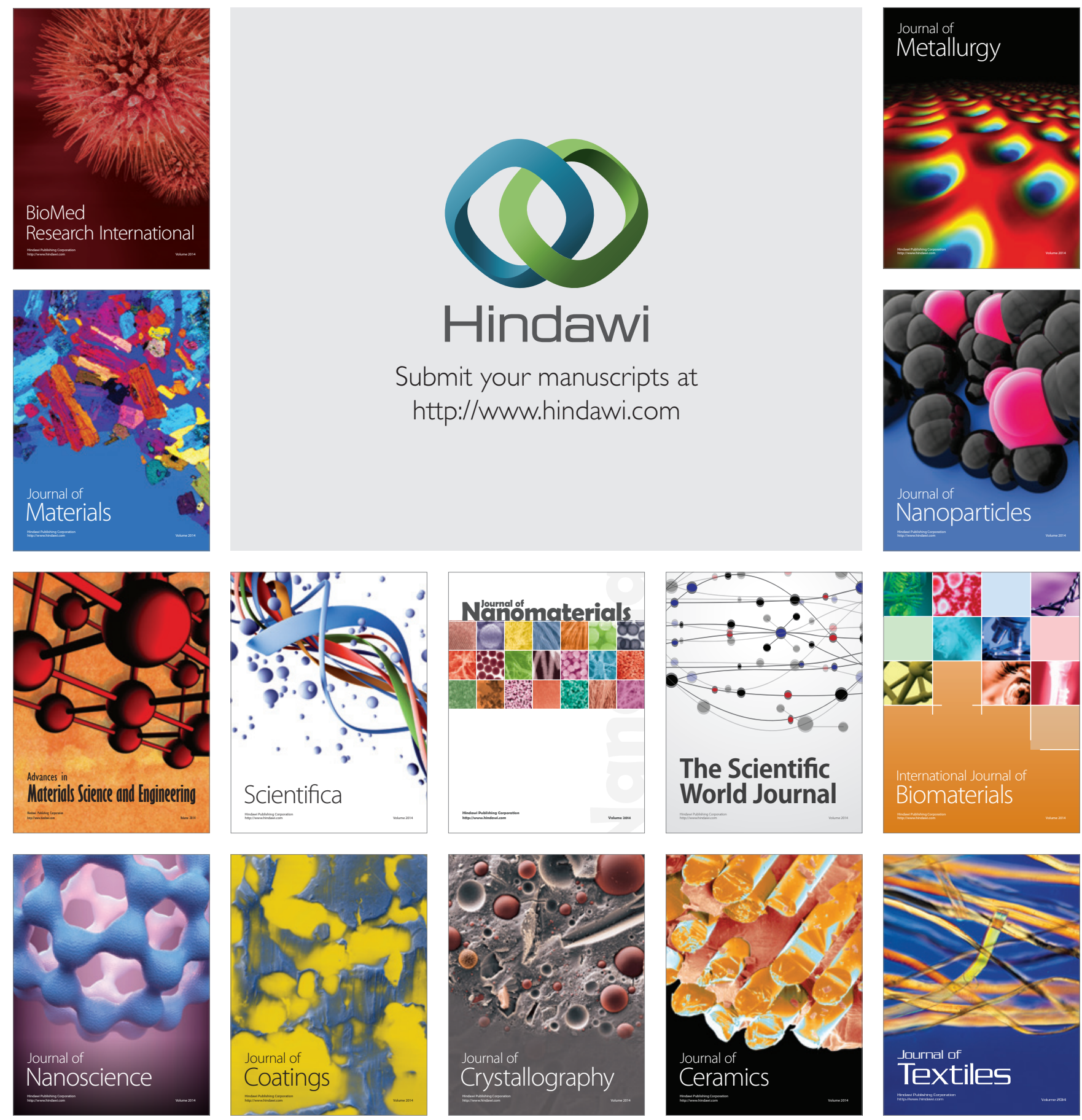